\title{
Meta
}

Journal des traducteurs

Translators' Journal

\section{Vidal Claramonte, Carmen África (2017) : "Dile que le he escrito un blues ", Del texto como partitura a la partitura como traducción en la literatura latinoamericana. Madrid : Iberoamericana/Vervuert, 186 p.}

\section{Danièle Marcoux}

Volume 64, numéro 2, août 2019

URI : https://id.erudit.org/iderudit/1068208ar

DOI : https://doi.org/10.7202/1068208ar

Aller au sommaire du numéro

Éditeur(s)

Les Presses de l’Université de Montréal

ISSN

0026-0452 (imprimé)

1492-1421 (numérique)

Découvrir la revue

Citer ce compte rendu

Marcoux, D. (2019). Compte rendu de [Vidal Claramonte, Carmen África (2017) : « Dile que le he escrito un blues », Del texto como partitura a la partitura como traducción en la literatura latinoamericana. Madrid :

Iberoamericana/Vervuert, 186 p.] Meta, 64(2), 562-565.

https://doi.org/10.7202/1068208ar d'utilisation que vous pouvez consulter en ligne. 
la matière du cinquième chapitre, sous la forme de l'analyse du projet de terminologie: les critères de sélection des sources et des termes en fonction des besoins, la conception et la confection de la fiche, la justification des différents champs. Le sixième et dernier chapitre («un réseau entre les connaissances») reprend les thématiques liées à l'organisation des connaissances évoquées dès l'introduction, pour aborder des questions importantes de politique linguistique. Partant du principe que les connaissances naissent dans les langues dans lesquelles elles prennent forme, l'auteure souligne l'intérêt pour la science de pratiquer une recherche plurilingue appuyée sur des terminologies fiables et également plurilingues.

Comme il se doit dans un manuel de type encyclopédique, l'auteure privilégie le socle commun des principes et des méthodes de la terminologie, désormais reconnue comme discipline. L'approche est donc consensuelle, ce qui n'empêche pas d'approfondir de nombreuses thématiques évoquées ni d'aborder les questions qui fâchent. Parmi ces dernières, citons un certain nombre de présupposés qui ne sont pas universellement acceptés, comme le pari que les connaissances se trouvent et peuvent être extraites des textes par le biais des termes. Quant aux points à approfondir, ils sont légion: c'est la fonction même d'une introduction d'aiguiller le débutant sur d'autres découvertes et de l'inviter à mener plus loin les réflexions amorcées au fil des pages. La question de la traduction revient dans tous les chapitres selon l'angle d'analyse retenu, c'est un des points forts du manuel. On aimerait poursuivre la discussion en s'interrogeant sur la traduction non pas des textes mais des termes. Le manuel nous indique comment identifier l'équivalent (ou les équivalents) d'un terme dans un texte dans les cadres de la traduction et de la terminographie. Mais que faire lorsque le terme posant problème est un néologisme dans les textes sources et que l'équivalent est introuvable? C'est une des questions que le lecteur se pose en lisant la section 2.5 , et le texte donne à réfléchir.

Résumons quelques qualités de ce livre. Constatons tout d'abord que son ambition encyclopédique se révèle parfaitement fondée: en l'espace de moins de 120 pages, l'auteure passe en revue l'essentiel des écrits et des réalisations en terminologie de ces cinquante dernières années. La bibliographie est riche, plurilingue (mais comportant d'importantes publications italiennes peu connues par ailleurs) et tout à fait à jour. C'est un livre qui est en prise directe avec les activités terminologiques internationales et leurs acteurs; il réserve une place importante aux nombreuses recommandations en terminologie en particulier de l'ISO. En même temps, l'étude est fermement ancrée dans la réalité italienne. Ce livre servira notamment à orienter le rôle de la terminologie dans le débat qui fait rage en Italie autour de la politique du «tout anglais» de certains milieux politiques et universitaires. C'est en effet un livre qui reconnaît l'intérêt de ne négliger ni la dimension diachronique ni les aspects culturels de la terminologie; c'est aussi un livre qui aborde directement la question de l'italien en tant que langue scientifique et technique.

JoHn Humbley

Université Paris Diderot, Paris, France Università di Verona, Vérone, Italie

\section{RÉFÉRENCES}

Adamo, Giovanni et Della Valle, Valeria (2017): Che cos'è un neologismo [Qu'est-ce qu'un néologisme]. Rome: Carocci.

ConCEIÇÃo, Manuel Célio (2005): Concepts, termes et reformulations. Lyon: Presses universitaires de Lyon.

Rey, Alain (1979/1992): La terminologie: noms et notions. Paris: Presses universitaires de France.

SCARPA, Federica (2001): La traduzione specializzata. Lingue speciali e mediazione lingüística [La traduction spécialisée. Langues de spécialité et médiation linguistique]. Milan: Ulrico Hoepli.

Zanola, Maria Teresa (2014): Arts et métiers au XVIII siècle - Études de terminologie diachronique. Paris: L'Harmattan.

Vidal Claramonte, Carmen África (2017): "Dile que le he escrito un blues", Del texto como partitura a la partitura como traducción en la literatura latinoamericana. Madrid: Iberoamericana/Vervuert, $186 \mathrm{p}$.

Pour l'entendre, il vous faut quitter la Plaza Mayor et prendre plutôt le passage piéton. Une fois vos pas posés dans les interstices de ce passage zébré, il surgit alors dans sa tenue bariolée, bigarrée, rapiécée. Tendez bien l'oreille: c'est Arlequin qui vous invite à parcourir avec lui l'essai de Carmen África Vidal Claramonte intitulé "Dile que le he escrito un blues", Del texto como partitura a la partitura como traducción en la literatura latinoamericana. Le patronage d'un tel personnage vous inquiète? Vous craignez que cet outsider par excellence, qui incarne le mélange, l'hybridité, voire l'incohérence, vous perde dans les dédales de la promenade proposée? Vous avez tort. Pour ouvrir la traduction à de nouveaux horizons conceptuels dans le contexte hybride de la mondialisation, pour l'aborder comme une activité hétérotopique à cheval sur des espaces et des temps épistémologiques variés, pour démontrer son rôle fondamental dans la transversalité des arts et leur 
fascinante interdisciplinarité, Vidal Claramonte ne peut compter sur meilleur accompagnateur qu'Arlequin.

Guidée par ce sympathique marginal, dont le costume illustre à merveille le corps métis du traducteur, l'auteure nous convie ainsi à une promenade des mots dans les mondes de la philosophie, la littérature, la peinture et, bien sûr, la musique. Le prologue signé David Johnston, mais ouverture conviendrait mieux peut-être tant il annonce le «texte partition», donne le ton sur lequel la professeure de l'Université de Salamanque module ses fines perceptions de la traduction: quand le traducteur sort les mots pour une promenade entre les langues et les cultures, ce qu'il rencontre n'a rien d'une ligne fixe parmi des sens mis à nu, mais tout plutôt d'une cartographie provisoire de l'aventure complexe qu'est la vie. Pour le lecteur, la promenade se transforme dès lors en une démonstration à la fois conceptuelle et méthodologique de la traduction vécue comme une activité de «décentrement " (Meschonnic 2009: 30) ou de «centre qui se déplace» (Blanchot 1955/2002: s. p.). Sur sa route, il croise un nombre impressionnant d'artistes et de penseurs non seulement contemporains, mais également vieux comme le monde, dont Aristote et Platon. Pour nombreuses qu'elles soient, ces rencontres n'occultent jamais l'horizon ou le but de la promenade, c'est-à-dire celui de montrer que les mêmes notions - bruit, son, rythme - traversent les domaines artistiques et contribuent à les définir avec, pour conséquence, la mise en évidence du rôle créateur et transformateur de la traduction. De la même manière qu'il faut passer par un autre mot pour découvrir la signification d'un mot, l'auteure propose donc, tout au long des cinq chapitres qui composent le livre, de passer par l'interdisciplinarité pour mieux définir la traduction. Belle balade en perspective!

Dans le premier chapitre, Vidal Claramonte s'appuie d'abord sur la vision ample et élargie de la traduction proposée par certains auteurs contemporains, ce qui lui permet ensuite de creuser son propre sillon, soit les affinités qu'elle observe entre l'interprétation d'un texte et celle d'une partition musicale. Digne héritière d'une lignée de penseurs pour qui la traduction est mouvement, «croisant des espaces et forgeant de nouveaux topoi à partir de loci connus $»^{1}$ (Johnston 2013:367), activité non seulement entre les langues, mais aussi à l'intérieur de celles-ci (Munday) ou ouverture des frontières disciplinaires (Tymoczko 2007), elle partage avec eux leur vision décentrée et aterritoriale qui définit le sens comme un «devenir» (Deleuze et Guattari 1968) ou une "force critique» impliquant une responsabilité face à l'art (Steiner 1976). De ce point de vue, tout texte s'aborde comme un territoire en construction, une partition ou une toile, où l'inter- prète utilise divers fragments pour reconnaître en eux des mondes différents qui interagissent avec des matériaux de nature fort diverse (Benjamin 1955/1988). Intéressant à signaler, Vidal Claramonte se base également sur ce que Platon appelait le melos, soit la synthèse du mot, de la tonalité et du rythme, pour illustrer le rôle de la musique dans ce phénomène d'extrapolation entre différents univers. Elle en donne de nombreux exemples: Kandinsky dont la recherche du rythme en peinture provient de la musique; Mallarmé affirmant que les mots établissent préalablement les relations créées par la musique; la technique du contrepoint musical mise au profit de l'écriture chez James Joyce, Aldous Huxley, Albert Camus, etc. En traduction, son approche lui permet de définir l'interprète telle la personne qui écoute (escucha) derrière la porte - métaphore de l'obstacle du langage - mais sans vraiment entendre (oír), au sens de comprendre, qui exerce l'art de la fugue en ne captant que des fragments de l'original, qui doit récrire moins le sens de l'original que la mélodie en contrepoint. Si la musique est présentée au sens très large de rythme entre les relations, Vidal prévient cependant que la vitesse de croisière, elle, varie forcément en fonction des interprètes, d'où le titre de ce premier chapitre: Allegro ma non troppo. Comparant des thèmes, des idées, et adoptant comme fil conducteur ce concept d'interprétation musicale, Vidal Claramonte y montre donc comment les disciplines artistiques se font écho les unes les autres, en mettant en lumière leurs rapports de traduction: s'ouvre ainsi la possibilité de dresser un parallèle entre les études traductologiques et le rôle privilégié de l'interprétation musicale dans la compréhension des rapports politiques, culturels, sociaux, une partition que maestro Vidal exécute avec doigté dans la suite du propos.

La négation du silence, l'affirmation des bruits, des rythmes, leur valeur pour la traduction assurent l'enchaînement et font l'objet du deuxième chapitre. S'inspirant des travaux de Michel Serres (1983), l'auteure définit le «bruit» comme la multiplicité inhérente à la parole, soit les multiples voix qui constituent le sens de chaque mot. Il s'agit ici d'un concept positif, le bruit nous rendant conscients de l'asymétrie et du déséquilibre qui existent entre les sens d'un même mot au sein de cultures différentes. Pour Vidal Claramonte, l'œuvre romanesque et poétique de John Cage ${ }^{2}$ illustre à la perfection la véritable nature des mots: ceux-ci ne surgissent jamais du silence ni de manière isolée, pas plus qu'ils ne sont neutres, ni qu'il existe de réalité sans langage. Tels les bruits que nous produisons, quand nous traduisons, les mots choisis nous trahissent, d'où le devoir du traducteur de réfléchir à la façon dont il construit le monde avec des mots, aux retombées 
éthiques de ses choix linguistiques, aux présupposés ontologiques sur lesquels se fondent ces choix. La manipulation à laquelle le langage nous soumet, les réécritures du texte original, les questions soulevées font du bruit, beaucoup de bruit. À l'ère de la mondialisation, Vidal Claramonte rappelle à quel point traduire, c'est jouer d'un puissant instrument capable de transformer la pensée ou, au contraire, de maintenir l'ordre établi. Chaque traducteur apporte ses bruits et ses rythmes en fonction de sa culture et de son idéologie, lesquels mettent en lumière l'hétéroglossie du texte, de la toile ou de la partition. Pour autant, il doit chercher les bruits et les rythmes contenus dans le texte - souvent couverts par nos propres rythmes - et différents des nôtres. Ce sont ces «musiques" que le traducteur doit récrire. Pour y parvenir, il doit prêter au texte une oreille attentive.

De concert avec les théories contemporaines de la traduction, qui reconnaissent le rôle clé de l'interprète dans le processus traductologique, notre spécialiste des rapports entre culture et idéologie propose ensuite de montrer, au chapitre trois, que le «concept d'équivalence» traverse toutes les disciplines artistiques (littérature, peinture, musique, etc.) et que son évolution s'est faite parallèlement dans tous ces domaines. Ici encore, ses réflexions s'articulent principalement autour de la musique et de la traduction. Vidal Claramonte affirme que les questions soulevées par le rôle de l'interprète, en musique, et du traducteur, en traduction - c'est ce qu'elle entend par concept d'équivalence -, sont les mêmes: l'interprète ne se borne pas à reproduire un texte ou une partition originale, soi-disant parfaite ou intouchable. Pour mettre en relief la subjectivité, la réécriture ou la recréation qui gouvernent l'interprétation, elle oppose les positions d'Igor Stravinski et Milan Kundera, qui prenaient leurs interprètes pour de simples transmetteurs invisibles de l'auteur, et celles de Glenn Gould et Jorge Luis Borges, qui les considéraient au contraire comme des créateurs ou des écrivains à part entière de l'œuvre. Elle commente amplement d'autres exemples de la deuxième approche, qui brouille les frontières entre auteur et traducteur, œuvre première et œuvre seconde, dont les célèbres Variations de Bach interprétées par Gould, le non moins célèbre Pierre Ménard, autor del Quijote de Borges ou, plus modestement, la traduction de Suzanne Lotbinière-Harwood du roman Le désert mauve de Nicole Brossard. Fidèle à cette vision ouverte et multiple de l'interprétation, Vidal Claramonte mentionne enfin que la littérature expérimentale, Larva de Julián Ríos, Alphabetical Africa de Walter Abish ou La disparition de Georges Perec par exemple, se prête particulièrement bien à cette approche. Il s'agit de textes qui «se recréent dans l'espace ludique des signifiants multiples, l'indétermination et l'ambiguïté et qui, pour autant, mettent en reliefl'inutilité ou l'absurdité de vouloir réduire les possibilités du sens à la cohérence ou à l'unité $»^{3}$ (p. 93). Dans ces circonstances, il est tout aussi illusoire de considérer la traduction comme un simple miroir qui refléterait l'original, puisqu'elle agit plutôt comme un prisme qui nous renvoie le spectacle subjectif de notre moi ou encore, comme le dit Vidal Claramonte en reprenant à son compte les mots de Steiner dans Langage et silence (1976), une sorte de miroir vivant, une contrepartie vitale, où traduire devient un acte amoureux. Mais avant d'aborder cet aspect, auquel l'auteure donne libre cours à la fin du livre, le quatrième chapitre vaut vraiment le détour.

Point culminant de la promenade proposée en ouverture, ce chapitre offre effectivement au lecteur de puissantes démonstrations de la possibilité de fonder une pratique de la traduction sur le contrepoint, ce procédé musical qui donne à entendre, simultanément, le chant d'une mélodie et celle d'un autre chant ou de plusieurs autres chants, tout en les maintenant sur un pied d'égalité. Si le procédé du contrepoint a été largement exploité en littérature, le corpus étudié par Carmen Vidal Claramonte se révèle particulièrement approprié pour analyser les nombreux défis de traduction soulevés par ces œuvres qui, selon Edward Said, mettent en lumière des rapports de pouvoir et d'asymétrie. L'impressionnant chœur d'écrivains et d'écrivaines rassemblés par l'auteure, Esmeralda Santiago, Julia Alvarez, Rosario Ferré, Tato Laviera, Luis Rafael Sánchez (Puerto Rico), Ana Lydia Vega, Cristina García (Cuba) ou Cecilia Vicuña (Chili), pour n'en nommer que quelques-uns, laisse ainsi entendre, en contrepoint, le métissage, le déplacement, l'instabilité et l'hybridation des voix qui composent leurs œuvres. Dans ce joyeux mélange d'influences linguistiques et culturelles, où les "watchar, tique pa'l cine, madamo» disputent aux «relajo, bachata, joda» le championnat des difficultés de traduction, le centre d'attention se déplace lui aussi du visuel vers l'auditif avec, en prime, une double utilité sur le plan de l'entendement des rapports entre langues, idéologies et cultures. Utilité méthodologique, d'abord, le contrepoint et ses stratégies de composition révélant la nécessité de réfléchir au sort passé et futur de toutes ces voix réduites au silence par l'impérialisme, aujourd'hui comme hier; utilité politique, ensuite, le contrepoint libérant la voix de ceux qui, dans le monde d'aujourd'hui, n'en ont pas. En misant sur le contrepoint ou sur la convivialité des voix, ce qui équivaudrait selon elle à jouer la même partition mais dans une autre clé, Vidal Claramonte oriente donc l'exercice de la traduction du côté de «l'hospitalité linguistique» 
(Ricœur 2004), en soulignant, du même coup, l'énorme responsabilité éthique qui incombe au traducteur, le premier à écouter la vie des autres et à la rendre possible... ou pas. Tel est l'objet du cinquième et dernier chapitre.

Revenant sur les lieux de l'hypothèse qui traverse tout le livre, à savoir que les mots ne se contentent pas de signifier mais qu'ils évoquent aussi des mélodies, des rythmes, des bruits, des textures, des saveurs, des odeurs, l'auteure libère toute sa subjectivité. Quand on touche les mots, dit-elle, les doigts restent imprégnés des impuretés des langues, dans cet espace de rencontre entre une langue et une voix, et où le grain de la voix signifie écouter notre relation avec le corps qui chante. Une relation érotique. Esquivant avec sensibilité le danger de succomber à une métaphysique du signe ou encore à une métaphorisation abusive de la traduction comme relation amoureuse, elle réfléchit plutôt concrètement au potentiel que recèle le traduire au moment de rendre audible à l'oreille la sagesse qui pense, à la connaissance quand elle se fait musique. D'où le lyrisme, certes, qui se dégage de cette déambulation à voix haute, mais dont les vibrations, jamais, ne font fausser l'ensemble. Parce qu'elle se lance à plein corps depuis la hauteur des mots, Carmen Vidal Claramonte doit être entendue. Sa voix singulière résonne dans notre mélodie une et commune. Si vous la croisez de nouveau à un passage zébré au bras d'Arlequin, dites-lui qu'elle nous a écrit un beau, un langoureux, un émouvant blues.

DANIÈle MARCoUX Université Concordia, Montréal, Canada

\section{NOTES}

1. Ma traduction.

2. CAGE, John (1961/2009): Silence. Lectures and Writings. Londres/New York: Marion Boyars.

3. Ma traduction.

\section{RÉFÉRENCES}

Benjamin, Walter (1955/1988): Dirección única. (Traduit de l'allemand par Juan J. Del Solar et Mercedes Allendesalazar) Madrid: Algaguara.

BlanCHOT, Maurice (1955/1988): L'Espace littéraire. Paris: Gallimard.

Deleuze, Gilles et Guattari, Félix (1968): Différence et répétition. Paris: Presses universitaires de France.

Johnston, David (2013): Professing Translation. The Acts-in-Between. Target. 25(3):365-384.

Meschonnic, Henri (2009): Éthique et politique du traduire. Paris: Verdier.

RICEUR, Paul (2004): Sur la traduction. Montrouge: Bayard.

Serres, Michel (1983) : Noise. SubStance. 12(3):4860.
Steiner, Georges (1976): Langage et silence. Paris: Les Belles Lettres.

Түмосzко (2007): Enlarging Translation, Empowering Translators. Manchester: St. Jerome.

Nord, Christiane (1997/2018): Translating as a Purposeful Activity: Functionalist Approaches Explained. $2^{\mathrm{e}}$ éd. Abingdon/New York: Routledge, $153 \mathrm{p}$.

Christiane Nord, grande ambassadrice de la tradition fonctionnaliste allemande, propose la deuxième édition de son célèbre Translating as a Purposeful Activity, ouvrage originellement publié chez St. Jerome en 1997. Fait à noter, la réédition paraît chez Routledge, au sein de la collection «Translation Theories Explored» dirigée par Theo Hermans. Les réalités de la traduction comme celles de la traductologie ayant passablement évolué au cours des vingt dernières années, il nous tardait de voir quelles nouveautés nous réservait cette réédition du livre de Nord.

D’emblée, mentionnons les deux principaux ajouts à l'ouvrage: en ouverture, un avant-propos signé Georges L. Bastin et, en clôture, un nouveau chapitre offrant un état des lieux du fonctionnalisme au $21^{e}$ siècle. Outre ces deux nouvelles parties, de nombreuses modifications ont été apportées tantôt globalement, tantôt ponctuellement.

Au chapitre des changements «mineurs», on retrouve notamment l'adoption de l'orthographe britannique (p. ex.: analysed, et non plus analyzed [p. 4]), l'amélioration de l'idiomaticité et du style (p. ex.: similarity to, au lieu de similarity with [p. 6]) et la correction de coquilles (p. ex.: Margret Ammann, plutôt que Margaret Ammann [p. 119]). En outre, au fil de l'ouvrage, Nord met à jour les références ou les remplace par de nouvelles lorsqu'opportun. Ces révisions sont toutes les bienvenues, mais mentionnons toutefois l'étonnant remplacement du terme languaculture par linguaculture, sachant que Nord fait uniquement appel à ce concept par le truchement des travaux de l'anthropologue Michael Agar qui affirme luimême que, lorsqu'il a emprunté le terme au collègue Paul Friedrich, il l'a fait passer de linguaculture à languaculture, "to bring it in line with the more commonly used "language" " (Agar 1994: 265).

En ce qui concerne les modifications "majeures», mentionnons d'abord la retraduction en anglais de quelques citations, une opération qui permet notamment de rectifier un glissement de sens présent dans l'ouvrage original. En effet, Nord y citait un extrait de Stylistique comparée du français et de l'anglais, à savoir «le plus petit segment de l'énoncé dont la cohésion des signes est telle qu'ils ne doivent pas être traduits séparément» (Vinay 